\title{
Weibo "Super Topic Community": Virtual Community from the Perspective of Interactive Ceremony Chain
}

\author{
Xiaozhen $\mathrm{Ma}^{1}$, Xiaojie Gong ${ }^{1}$, Xiaofeng Cong ${ }^{1}$ and Jia Cong ${ }^{2, *}$ \\ ${ }^{1}$ School of Political Science and Law, University of Jinan, Jinan, Shandong 250022, China \\ ${ }^{2}$ Marketing Department, Lancaster University, City of Lancaster LA14YX, The United Kingdom \\ *Corresponding author Email: 2428407729@qq.com
}

\begin{abstract}
The purpose of the study is to substitute the theory of interactive ritual chain to analyze the interaction logic, characteristics, rules and motivations of the members of the microblog "Super Topic Community", and discuss its possible construction results and practical problems. This electronic uses participatory observation and in-depth interviews as research methods, and takes the current popular "Langlang Ding" Super Topic Community as a research sample, ten samples were drawn from it as research objects. In the end, the study found that interest needs and emotional satisfaction are the starting point and motivation of member interaction rituals. The interpersonal relationship between members within the community is a "net-born" relationship construction process, forming a "circle-oriented" relationship structure and field pattern. Interactive features. And through the established rules to restrain members, gradually internalized into members' self-restraint, weaken the subjectivity of individual individuals, strengthen group consciousness, and build group identity.
\end{abstract}

Keywords: virtual community; super chat on Weibo; interactive ceremony; interaction logic

\section{THEORETICAL BASIS AND LITERATURE REVIEW}

At present, domestic and foreign scholars' research related to the theory of "virtual community" and "interactive ritual chain" involves multiple fields, multiple topics, and multiple directions, and the research results are also very rich.

\subsection{Theoretical Research on Virtual Community}

When foreign scholars define virtual communities, they focus on the core elements of four aspects: people as the main body, interaction as the motivation, the Internet as a tool, and the connection of interpersonal relationships as attributes. In 1993, the American scholar Howard Reynolds first proposed the concept of "virtual community" in his book "Virtual Community-Home of Electronic Territory". He clearly delineated the community aggregation attribute of "virtual community", and proposed that virtual community is a network of interpersonal relationships formed by a group of people who share common emotions through long-term public discussions through computer networks.

In my country, scholars in different disciplines have defined the concept of virtual communities in multiple dimensions: Jia Erpeng and Chen Jianxin (2011) pointed out in their research that virtual communities are essentially micro-regions or spaces, which are gathered and owned by social websites in the network environment. A community of common needs and interests. [1] Cai Qi and Yue Lu (2018) believe that: "Internet virtual communities are a collection of communities based on a certain network application platform for interpersonal communication and interaction." [2] She Shuo and Nie Huijing (2018) believe A virtual community is a social media platform for groups with common interests and goals to regularly communicate and interact with each other on the premise of trust and recognition in accordance with established rules. [3]

Based on the opinions of most scholars at home and abroad, this article believes that virtual communities are network activities that rely on information technology to deepen the common emotions, interests and interests of community members through interactive communication under established rules.

\subsection{Interactive Ritual Chain Theory}

The concept of "ritual" first appeared in Durkheim's sociology of religion. It was Owen Goffman who really proposed the concept of "interactive ritual". In his work, he mentioned that interactive rituals are embodied in all aspects of people's daily communication and proposed Another important thesis is "co-presence", that is, members must interact with a physical space within the same period of time to be effective communication behavior, in order to have an impact and resonance with other members in the same situation. [4] 
Based on the knowledge of Durkheim and Owen Goffman, Collins generalized and expanded the interactive ritual chain theory. [5] In addition to recognizing that interactive rituals should include value resonance and common presence, he also emphasized that the process of interactive rituals shields outer personnel who do not meet the standards, and that the consistency of needs and values of inner personnel in a common situation unfolds to the same point of interest or Discussing the focus of attention such as interests, and excluding members that do not meet the definition of the circle can maintain the stability of the internal structure and maximize the interests of the circle members.[6]

Based on the viewpoints of the above-mentioned scholars, the interactive ritual chain contains four main points: first, members need to have common value standards and emotional needs; second, members pay attention to the focal points and topics with high homogeneity; third, the division of member groups is determined by Standardly, inner members will restrict outer members according to established rules: Fourth, the interactive ceremony requires members to be in the same situation to influence each other.

\subsection{Weibo "Super Topic Community": Fans Gather in the camp}

Super Topic Community is an interactive organization of online interest content gathered by members with the same fun-related culture based on a topic of sustainable discussion.

Generally, members of the Super Topic Community are celebrity fans. Fans create or browse various types of posts in Community based on their love and attention to idols.The Topic Community carries the function of expressing emotions and connecting and interacting with fans. Reach a consensus: that is, there will be no excessive large-scale discussion and interaction outside the community, so as to prevent negative effects on their idols. However, internal interactive discussions will further deepen their feelings for idols and may form a small interactive circle, so the community has become a gathering camp for fans.

\section{RESEARCH METHODS AND RESEARCH SAMPIES}

\subsection{Participatory Observation Method}

The author has participated in the topic discussion of "Langlang Ding" Super Topic Community for three months. During this period, I have been following various fan activities in there. A total of 96 posts were released, with a maximum reading of 216,000 . The number of single Weibo comments reached 270 times and the number of likes reached 466 times. At the same time, the author will also browse and follow relevant fan updates on multiple platforms such as station B, LOFTER, and QUOTEV.

\subsection{In-depth Interview}

This article selects ten representative community members as the in-depth interview subjects, including management members, active fans, and ordinary fans to learn about their interaction modes, interaction characteristics, and interaction characteristics through Weibo private messages. Interaction rules, interaction motives and final interaction results are several modules, exploring the interaction logic of members in the special virtual community and the positive and negative results behind the logic.

\subsection{Research Samples}

The research sample of this article is the "Langlang Ding" community, and the fans of the current hot traffic stars Gong Jun and Zhang Zhehan are gathered in the Super Topic Community. The community currently ranks first in the CP community category of the Weibo Super Topic Community section. It can be seen that its related topics have a high degree of popularity and attention. There is a large amount of interaction in the community, and the interactive content is rich and diverse. It has only experienced four. The monthly development has a stronger order and a more complete internal structure, and the data, anti-criminal, output, resource provision and other team departments are all available, and have research references. This study selects 10 members from the "Langlang ding" community as the in-depth interview samples. Among them are the community administrators, and the grainproducing wives who have videos, writing and drawing are full-time or part-time creative members. There are also active fans with high enthusiasm for interactive behaviors such as posting, commenting, and liking, and ordinary fans with low participation.

Table 1 In-depth interviewees

\begin{tabular}{|c|c|c|c|}
\hline $\begin{array}{c}\text { Nickname } \\
\text { Occupation }\end{array}$ & $\begin{array}{c}\text { Age } \\
\text { (years } \\
\text { old) }\end{array}$ & Occupation & Gender \\
\hline $\begin{array}{c}\text { Little soil } \\
\text { turtle }\end{array}$ & 31 & civil servant & male \\
\hline $\begin{array}{c}\text { Call me Zou } \\
\text { Yu }\end{array}$ & 22 & Freelance & Female \\
\hline $\begin{array}{c}\text { Tao Tao Bo } \\
\text { Bo Bing }\end{array}$ & 18 & student & Female \\
\hline $\begin{array}{c}\text { Wood food } \\
\text { Mushroom } \\
\text { shore }\end{array}$ & 29 & $\begin{array}{c}\text { Self-media } \\
\text { blogger }\end{array}$ & Female \\
\hline Meow Daxian & 25 & $\begin{array}{c}\text { Self-media } \\
\text { blogger }\end{array}$ & Female \\
\hline Yokun & 23 & student & Female \\
\hline
\end{tabular}




\begin{tabular}{|c|c|c|c|}
\hline Chiba & 23 & $\begin{array}{c}\text { Members of } \\
\text { Foreign }\end{array}$ & Female \\
\hline Junhan & 17 & student & male \\
\hline $\begin{array}{c}\text { From } \\
\text { Xingyue }\end{array}$ & 18 & student & Female \\
\hline
\end{tabular}

\section{LOGIC OF FAN INTERACTION BEHAVIOR IN THE "SUPER TOPIC COMMUNITY"}

In the Super Topic community, the common focus of fans' attention is the celebrity's dynamics and related topics. The occurrence of communication and interaction behaviors is also based on common interests and emotional needs. Only with this interaction motivation can real interaction behaviors be triggered. Communities with different topics of interest will attract fan members from different circles, and the interpersonal relationship between fans is also a process from weak to strong, presenting a "net-born" interpersonal relationship development model.

\subsection{Interactive Mode: "Internet-based" Communication Mode}

The establishment of interpersonal relationships relies on specific opportunities and bonds. The information in the virtual community is relatively concealed. The members are no longer in strong connections, but instead support a weak connection with "interesting relationships". The establishment of community has a certain fan base. The process of increasing the number of fans reflects the development process of interpersonal relationships from weak to strong. This is also the first path of relationship development in cyberspace.

"I just came in and browsed through the posts, and now I will urge you to produce a lot of food, and I will often comment on private messages." "For offline events, go to the physical store to buy endorsements. I have met many fans like me. " (Interviewee: Mushroom shore)

"Slowly private comments are really bombastic, but I also added a lot of contact information of bloggers I like very much." "The mountain people are really kind and lovely. I have participated in offline activities, which was in early May. Those two concerts." (Interviewee: Wood food)

Therefore, the "net-born" interpersonal relationship in the virtual space is constantly deepening with the accumulation of interaction and communication. On the one hand, the "net-born" interpersonal relationship can be understood as a relationship formed by people who have nothing to do with each other through the Internet on the basis of virtual imagination. On the other hand, strong relationships after being stabilized will also become tools for the expansion and extension of new interpersonal relationships, consolidating the community and also driving the development of new weak relationships.
"In the comments, there will be situations where new fans won't read them. Generally, I don't pay much attention anymore, because there are too many replies, and if there is something that I'm not sure about, the other mountain people in the comment area will answer. Many fans retweeted my article, which attracted more fans' attention." (Interviewee and Jun)

On the whole, the construction of interpersonal relationships between community members is the process of building relationships from being unconnected in reality to forming weak relationships of tentative communication and then turning into a "net-born" relationship from reality to strong relationships; Strong ties will in turn promote the expansion of weak ties.

\subsection{Interactive Features: "Circling" Relationship Structure and Field Pattern}

Many researchers have proposed that the structure of a virtual community is a "circle" type with clear virtual boundaries. The division standard is set by the community manager. The purpose is to distinguish the members inside and outside the "circle". The most obvious feature is It requires identity authentication and will have its own name. In "Langlang Ding" Community, the internal members are collectively referred to as "Shanren", they will share text, video, audio and other forms of post content inside community, and they are not familiar with the topic of their concern outside the circle. He will not introduce and express indiscriminately, so as not to arouse the resentment of external netizens in the circle, and prevent discrediting his own celebrities. Of course, he will not easily set foot in other hyper-talking areas that have a clear sense of competition with the topic.

"Enclosure is good for being cute. If CP fans over dance outside, they must be black. In fact, there are still people who are not rational, and their CP acceptance is not that high." (Interviewee: Meow Daxian)

"Fan's account will comment and like, like you, and only promote it in front of friends around you. You can't just talk to others casually. It's too unrealistic. We don't necessarily like others. The whole set of chaos must be hacked." (Interviewee: Call me Zou Yu)

Communities based on different interests, interests, and beliefs will distinguish "outsiders" and "insiders", and conflicts will inevitably occur in a state of opposition. Of course, the inner circle will show a relatively harmonious and stable hierarchical structure, and the fan level can be divided into: core fans, active fans and ordinary fans. The fans in these circles will also be based on different discourse patterns and cultural practices. The categories show a level of difference in circles. 


\subsection{Interactive Rules: Sustainable Restraint Rules Recognized and Followed by Community Members}

Online virtual communities also need rules and systems to ensure the healthy operation of the community. The diversified external information received by community members cannot fully guarantee the positive effect on the development of the community. If it is not censored and restricted, it will cause internal instability. The Super Topic Community on the Weibo platform are managed by internal administrators, namely Community Hosts. The host group has the right to post the community publicity, shielding and clearing the content of posts that violate the publicity, or even forcibly removing internal members.

"I usually pay attention to the announcement information, because there will indeed be some posting or account problems, which is also convenient for us to actively fight against hacking and do data." (Interviewee: Little soil turtle)

"My post was screened, that is, the post could not be posted and thought it was a problem with Weibo or my phone. It turned out to be black out of my post, but I don't know why, and I didn't pay much attention to the announcement." (Interviewee: Meow Daxian)

The Super Topic Community established rules have a strong binding force on members, and most people will abide by them when they are enforced. They will gradually be internalized into members' self-discipline, weakening the subjectivity of individual individuals, strengthening group consciousness, and building group identity.

\subsection{Motivation for Interaction: Meeting Common Interests and Emotional Needs}

Various ritual activities in the virtual community will realize the exchange and satisfaction of interests when members exchange interests and transmit information, further enrich the emotional space, generate emotional resonance, and form a shared emotional state and identity. In the "Langlang Ding" super chat, although members will not be online in real time, the purpose of entering the community is very strong. It is to obtain dynamic information about Gong Jun and Zhang Zhehan and to understand the reactions of members of the same group after paying attention to the same information. Regardless of whether the reactions are similar or not, different excitement points can be stimulated, creating and connecting a kind of "emotional community".

"I was impressed by their acting skills and feelings. After entering the community, I can meet the sisters who can talk with each other. I added a lot of contact information and developed into real friends." (Interviewee: Wood food)

"When I see a lot of excellent works in community, I want to collect and save them. The authors will be very enthusiastic, and even many other fans who have not contacted will take the initiative to send me private messages. The relationship between everyone is really good." (Interviewee: From Xingyue)
Interest and emotion are both the starting point of member interaction and the motivation of the interaction ceremony. Both actually have the attributes of symbiosis. Interest triggers emotional resonance. In turn, strong emotion will stimulate stronger interest. This is formed in the process of interaction. Two-way interactive ritual chain.

\subsection{Interactive Results: Information Diffusion and Dissemination of the Homogenized Fun- related Culture}

The interactive space for fans is not limited to the Weibo platform. Fans will spread the content and information they pay attention to on different platforms through transportation and other forms. This realizes the interaction between the platform and the platform. It is also the simultaneous promotion of multiple platforms. Other fans with the same fun culture provide a channel of entry. When fans post relatively novel dynamics, they will arouse a wide range of discussions and attention, presenting a many-to-many information transmission mode in which individuals use social network as the path.

"I will often browse Tiktok, Douban, and Zhihu. There will be a lot of content I want to know, and the repetition rate is quite high. Many of them have been seen many times, but I will continue to browse, and many have never The content that you have contacted will be reposted to your account and shared with more people." (Interviewee: Tao Tao Bo Bo Bing)

"I usually share works that I think are very good with everyone. I will mark the author and link to make it easier for you to find. If you like it, you will save it directly or go to the original author to comment and save." (Interviewee: Chiba)

\section{CONSTRUCTION RESULTS AND REALISTIC ISSUES}

\subsection{Construction Result: the Connection of Interpersonal Network}

In interactive rituals, the common presence, the cultivation of common interests and emotions, and the sense of common identity have all become important elements of weaving a network of interpersonal relationships. In the virtual network community, the real interpersonal relationship is continuously reproduced into the virtual space. It also promotes the development of highly resonant emotional relationships offline, and realizes the crossfusion of virtual relationship networks and real relationship networks. For fans, in addition to discussing the main topics in Super Topic Community, they will also publish their daily life and feelings in order to obtain encouragement from others and transform them into motivation to support themselves. 
"I've followed that the living conditions of some sisters in the group are not very good. In fact, sometimes I feel a little uncomfortable when I see the posts they post. I will type what I want to say under the posts. I don't know if I can see them. But at the time I really want to say something to comfort her." (Interviewee: Yokun)

"I like to make friends. When I meet some interesting sisters, I will also communicate in private messages. Sometimes it's not just about discussing them. I may talk about some of my own situation. Everyone is very different in age, but they can get together. Encouragement is also very good." (Interviewee: Wood food)

The humanistic care between fans has become the glue of interpersonal relationships, making the relationship between each other closer, and building a network of interpersonal relationships in circle interaction.

\subsection{Realistic Issues: "Alienation" of Values in Member Interaction}

Information on the network platform is highly fluid and mixed, and members' information reception is one-sided and more easily affected by various types of information, and there will inevitably be conflicts with biased thoughts. The lack of a safety valve system for fans to vent in cyberspace, coupled with the concealment of personal information in cyberspace and the high degree of freedom provided by the platform, often allows some loose groups or irrational fans to take advantage of them, deliberately instigate and negatively guide. This has led to frequent online violence, frequent behaviors and loss of rationality. Some fans are over-invested in chasing idols, and bad behaviors such as car following, proxy shooting, squatting seriously interfere with idols' daily life; some fans oversupport idol endorsements, excessive consumption without sufficient financial capacity, affecting their normal life . The control of the network platform is limited, and the anonymity system allows many members to speak freely, even maliciously. The linkage between fans makes this group agitate as soon as it reaches a certain scale. Extremist behaviors will affect other ordinary users on the platform, cause resentment, and are not conducive to the reasonable development and use of the platform. Therefore, excessive star-chasing and obsessive, irrational fans are not support for idols, but a deformed development of values.

\section{CONCLUSION}

The research in this paper finds that the interpersonal relationship between members of the community is a kind of "network-born" relationship construction process, which forms the interactive characteristics of the "circle-like" relationship structure and the field pattern, and the members are gradually restrained through established rules. Turn it into self-restraint of members, weaken the subjectivity of individual individuals, strengthen group consciousness, and build group identity. Meanwhile, interest needs and emotional satisfaction are both the starting point of interaction between members and the motivation of the interaction ritual. The two have symbiotic attributes. Interest triggers emotional resonance. In turn, strong emotions will inspire stronger interest. This is in the process of interaction. The two-way interactive ritual chain formed in the process ultimately realizes the information diffusion and dissemination of the homogenized fun-related culture.

Of course, in the process of construction, the community will also induce many practical problems due to factors such as the irregular system, the one-sided reception of member information, and the extreme nature of the fans themselves. The research space for hyper talk virtual space is still broad, and the research content in this article will be further improved as the understanding deepens.

\section{REFERENCES}

[1] Erpeng Jia and Jianxin Chen, Research on virtual communities at home and abroad[J]. New Century Library, 2011(12): 32-36. DOI: 10.16810/j.cnki.1672514x.2011.12.009 (In Chinese)

[2] Qi Cai and Lu Yue, The path, mode and value of the construction of interpersonal relationships in virtual communities on the Internet [J]. Modern Communication (Journal of Communication University of China), 2018, 40(09): 143-147+152. (In Chinese)

[3] Shuo She and Huijing Nie, The definition of the concept of virtual communities in my country and the visual analysis of research hotspots[J]. Library Theory and Practice, 2018(01): 15-20. DOI: 10.14064/j.cnki.issn1005-8214.2018.01.003 (In Chinese)

[4] Xiaolong Xu and Fanghua Wang. Research on the knowledge sharing mechanism of virtual communities[J]. Studies in Dialectics of Nature, 2007(08): 83-86. DOI: 10.19484/j.cnki.10008934.2007.08.019 (In Chinese)

[5] Dechao Kong. Research on the Knowledge Sharing Model of Virtual Community[J]. Research in Library Science, 2009(10): 95-97. DOI: 10.15941/j.cnki.issn1001-0424.2009.10.027 (In Chinese)

[6] Feirong Wang, Jianli Gong and Chai Jinying, Research on the Knowledge Sharing Operation Mechanism of Virtual Community[J]. Zhejiang Academic Journal, 2007(05):201-205. DOI: 10.16235/j.cnki.33-1005/c.2007.05.001 (In Chinese) 